\title{
Evolution of the magnetic field generated by the Kelvin-Helmholtz instability
}

M. Modestov, ${ }^{1}$ V. Bychkov, ${ }^{2}$ G. Brodin, ${ }^{2}$ M. Marklund, ${ }^{2,3}$ and A. Brandenburg ${ }^{1,4}$

${ }^{1}$ Nordita, KTH Royal Institute of Technology and Stockholm University, Roslagstullsbacken 23, SE-10691 Stockholm, Sweden

${ }^{2}$ Department of Physics, Umeå University, SE-901 87 Umeå, Sweden

${ }^{3}$ Department of Applied Physics, Chalmers University of Technology, SE-41296 Gothenburg, Sweden

${ }^{4}$ Department of Astronomy, Stockholm University, SE-10691 Stockholm, Sweden

(Received 14 February 2014; accepted 14 July 2014; published online 31 July 2014)

The Kelvin-Helmholtz instability in an ionized plasma is studied with a focus on the magnetic field generation via the Biermann battery (baroclinic) mechanism. The problem is solved by using direct numerical simulations of two counter-directed flows in 2D geometry. The simulations demonstrate the formation of eddies and their further interaction and merging resulting in a large single vortex. In contrast to general belief, it is found that the instability generated magnetic field may exhibit significantly different structures from the vorticity field, despite the mathematically identical equations controlling the magnetic field and vorticity evolution. At later stages of the nonlinear instability development, the magnetic field may keep growing even after the hydrodynamic vortex strength has reached its maximum and started decaying due to dissipation. ( 2014 AIP Publishing LLC.

[http://dx.doi.org/10.1063/1.4891340]

\section{INTRODUCTION}

The Kelvin-Helmholtz (KH) instability is one of the most important, fundamental, and powerful phenomenon in fluid mechanics and plasma physics. The instability develops at the interface between two fluids (gases, plasmas), when one component is gliding along the other. ${ }^{1}$ The most important outcome of the $\mathrm{KH}$ instability in nature is the generation of turbulence via cascades of interacting vortices. Various examples of the KH instability may be encountered in geophysical and astrophysical flows, from ocean surface waves exited by wind, turbulent jets, and wakes up to large-scales instabilities in the interstellar medium, accretion discs, and supernova remnants. ${ }^{2-4}$ In quantum gases, the KH instability may produce quantum turbulence of a qualitatively new type at the interface of two immiscible Bose-Einstein condensates. ${ }^{5,6}$ In combustion, powerful $\mathrm{KH}$ instability arises at the late stages of deflagration-to-detonation transition making the flow of burning gases strongly turbulent and facilitating generation of strong shocks. ${ }^{7}$ Presently, there is also growing interest in the $\mathrm{KH}$ instability in the context of inertial confinement fusion (ICF). ${ }^{8-12}$ Initially, the interest in the $\mathrm{KH}$ instability in laser plasmas has been fueled by research focusing on the Rayleigh-Taylor (RT) instability, which has been one of the most actively explored problems within the ICF applications for decades. At the nonlinear stage of the RT instability, light fluid (pushing or supporting a heavy one) forms bubbles rising "up," with spikes of heavy matter falling "down" in a real or effective gravitational field. ${ }^{13-16}$ The relative motion of light and heavy components results in a secondary $\mathrm{KH}$ instability with subsequent generation of turbulence and possible mixing of the two substances. The well-known mushroom structure of the RT bubbles is, in fact, the outcome of the secondary KH instability.

However, lately, a large number of papers have addressed the ICF related KH instability for its own sake without direct relation to the RT instability. ${ }^{8-12,17}$ A good deal of experiments have been designed and performed on the Omega Laser Facility focusing on the KH instability, e.g., at the foam-aluminum interface in a layered target with two different substances set in motion by counterpropagating shock waves. ${ }^{12}$ The other option was inducing the KH instability by a shock refracted at an interface separating two substances of noticeably different density. ${ }^{9-11}$ The purpose of these experiments was typically to study generation of vortices and turbulence at the $\mathrm{KH}$ unstable interface. There has also been much interest in the RT and KH hydrodynamic instabilities as sources of magnetic field in plasmas. Several mechanisms of magnetic field generation in plasmas have been proposed, including thermo-electric and baroclinic effects and the ponderomotive force from an inhomogeneous laser beam. ${ }^{18-21}$

Under extreme ICF conditions, plasma motion is expected to produce an ultra-high magnetic field, which may alter the plasma flow dynamics as well as influence background magnetic and electric fields. The earliest measurements of the magnetic field produced by laser plasma flows were made already in seventies, detecting kilogauss field strength. ${ }^{18,22}$ Modern powerful laser setups stimulate the experimental activity in this area during the last few years. Recent experiments on the RT instability at the OMEGA laser facility demonstrated generation of the magnetic field with values up to 1 MGauss. ${ }^{23-25}$ In order to obtain thorough knowledge of the $\mathrm{KH}$ instability phenomenon, a special setup has been designed and built within the OMEGA facilities. ${ }^{8}$ Unfortunately, so far, experiments on the laser-driven $\mathrm{KH}$ instability have been performed without direct measurements of the instability-generated magnetic field. ${ }^{8-12,17}$

Much work has also been done on numerical simulations of both the RT and KH instabilities, taking into account the resulting magnetic field generation. ${ }^{26-30}$ First numerical studies considered the Biermann battery mechanism within 
the astrophysical problems, searching for the origin of the protogalactic magnetic field. ${ }^{26}$ In the context of the ICF, the numerical simulations ${ }^{27-29}$ have investigated the magnetic field generation by the RT instability at an inert 2D interface, which is, however, a too oversimplified model as compared to the RT unstable laser ablation flow in the ICF. Simulations of the KH instability in laser plasma have also been performed, but they did not take into account the magnetic field generation. ${ }^{11}$ Only recently, magnetic field generation by the KH instability has been considered, ${ }^{30}$ although the study has been performed within the kinetic, not magneto-hydrodynamic (MHD) approach, for the specifically astrophysical cold-fluid KH perturbations and electronion shear flows. Thus, the study of magnetic field generation by the $\mathrm{KH}$ instability within the MHD approach has been required; the purpose of the present work is to compensate for the gap in the studies.

The purpose of the present paper is to investigate generation and evolution of the magnetic field arising from the $\mathrm{KH}$ instability due to the Biermann battery (baroclinic) effect in the characteristic MHD geometry of two counter-directed flows of conducting plasmas. The problem geometry is a natural setup for the KH studies similar to the Omega Laser facility experiments. ${ }^{8-12}$ Analytical treatment of the full set of MHD equations is extremely difficult due to the nonlinear terms, although linear stability analysis may provide necessary estimates for further experiments and computer studies. By contrast, direct numerical simulations (DNS) are a much more powerful tool, which provides a complete picture of the plasma dynamics. For this work, we have performed numerical simulations of the magnetic KH instability using the Pencil Code. ${ }^{31,32}$ First of all, we show that the KH instability does generate magnetic field due to a baroclinic term in the induction equation. We observe and discuss the dynamics of the generated magnetic field and the vorticity field in the flow. In contrast to previous studies of the RT instability with magnetic field generation, ${ }^{27-29}$ we show that the magnetic field and vorticity behavior in the flow may be qualitatively different. In particular, the magnetic field may yield complex structures influenced by secondary KH instabilities at smaller scales. Our simulations show that the magnetic field continues to grow even after the hydrodynamic vortex has been developed and started decaying due to non-zero plasma viscosity. The results obtained demonstrate that the relation between vorticity and the magnetic field in the MHD instabilities is not as straightforward, as it was believed previously, and indicate wide prospects for future research, including both experimental, theoretical and numerical approaches.

\section{THE BASIC PLASMA MODEL EQUATIONS AND THE NUMERICAL METHOD}

To study magnetic field generation owing to the $\mathrm{KH}$ instability, we solve the compressible MHD equations for a visco-resistive plasma that is fully ionized. The magnetic field is resolved in terms of the magnetic vector potential $\mathbf{B}=\nabla \times \mathbf{A}$, thus ensuring zero divergence of $\mathbf{B}$. The governing equations of plasma dynamics are

$$
\begin{gathered}
\frac{D \ln \rho}{D t}=-\nabla \cdot \mathbf{u}, \\
\frac{D \mathbf{u}}{D t}=-\frac{1}{\rho} \nabla p+\frac{1}{\rho} \mathbf{J} \times \mathbf{B}+\frac{1}{\rho} \nabla \cdot 2 \nu \rho \mathrm{S}, \\
\frac{\partial \mathbf{A}}{\partial t}=\mathbf{u} \times \mathbf{B}-\eta \mu_{0} \mathbf{J}+\beta \frac{1}{\rho} \nabla p, \\
\frac{D s}{D t}=\frac{1}{\rho T} \nabla \cdot(K \nabla T)+\frac{1}{\rho T} \eta \mu_{0} \mathbf{J}^{2}+\frac{1}{T} 2 \nu \mathrm{S}^{2},
\end{gathered}
$$

where $D / D t=\partial / \partial t+\mathbf{u} \cdot \nabla$ is the advective time derivative, $\rho$ is the plasma density, $\mathbf{u}$ is the velocity, $p$ stands for the pressure,

$$
s=c_{P} \ln \left(\frac{p^{1 / \gamma}}{\rho}\right)
$$

is specific entropy, $\mathbf{J}=\mu_{0}^{-1} \nabla \times \mathbf{B}$ is the current density, $\nu$ and $\eta$ are the kinematic viscosity and magnetic diffusivity, respectively, $K$ is the thermal conductivity, $\mathbf{S}$ is the strain tensor,

$$
\mathrm{S}_{i j}=\frac{1}{2}\left(\frac{\partial u_{i}}{\partial x_{j}}+\frac{\partial u_{j}}{\partial x_{i}}-\frac{2}{3} \delta_{i j} \nabla \cdot \mathbf{u}\right),
$$

$\beta=m_{\mathrm{p}} / e$ is the proton mass to charge ratio, and $T$ is temperature. The ideal gas equation complements Eqs. (1)-(4), so that the pressure is given by $p=\rho c_{s}^{2} / \gamma$, where $\gamma=c_{P} / c_{V}=5 /$ 3 is the ratio of specific heats at constant pressure and volume, respectively, the sound speed is a function of density and entropy determined for a polytropic gas as

$$
c_{s}^{2}=c_{s 0}^{2} \exp \left[\gamma s / c_{p}+(\gamma-1) \ln \left(\rho / \rho_{0}\right)\right],
$$

and $c_{s 0}$ and $\rho_{0}$ are normalization constants. The last term in the induction equation (3) is identical to the baroclinic term in the vorticity equation, see Eq. (12) below, which represents the Biermann battery mechanism of magnetic field generation. The Hall term has been omitted as it is important only in the case of extremely large magnetic fields, which are more probable for the RT instability rather than the $\mathrm{KH}$ instability. Moreover, even Mega Gauss field strength may not be enough for any significant influence of the Hall term as demonstrated in Ref. 28.

The set of Eqs. (1)-(6) has been solved with the help of the Pencil Code, ${ }^{31,32}$ based on sixth-order finite difference spatial derivative approximations and a third order RungeKutta scheme for time stepping. The code is primarily used to solve 3D problems, such as turbulent solar dynamo evolution in Cartesian or spherical coordinates. In addition, for efficient massive calculations, the code is parallelized in all directions using the message passing interface library. The time step is computed automatically depending on the advection speed, the viscosity as well as the magnetic and thermal diffusivities. No numerical viscosity is used other than the explicit one which dominates over that from the diffusive and dispersive discretization errors associated with the numerical scheme. ${ }^{38}$ If the resolution is insufficient for given viscosity, energy will not dissipate at the smallest scales, and 
the code will "crash," signaling then the demand for better resolution.

The upper and lower walls are assumed to be impenetrable stress-free boundaries (i.e., hypothetic walls with slip boundary conditions) with a perfect conductor conditions for the magnetic vector potential

$$
\begin{array}{ll}
u_{y}=0, & \partial u_{x} / \partial y=0 \\
A_{y}=0, & \partial A_{x} / \partial y=0
\end{array}
$$

In the other directions, we use periodic boundary conditions. In all the simulations presented below, we use $1152^{2}$ meshpoints.

The $\mathrm{KH}$ instability is essentially a $2 \mathrm{D}$ phenomenon, so that its main features may well be studied in two dimensions. Taking into account 3D geometry, one has to face turbulent mixing in the transverse direction to the initial flow plane. This may conceal important physical properties of the $\mathrm{KH}$ instability and makes it computationally expensive and harder to investigate. Moreover, considering magnetic fields in $3 \mathrm{D}$, there is inevitably magnetic dynamo action, which affects the evolution of the magnetic field. ${ }^{33}$ In a $2 \mathrm{D}$ flow, magnetic fields originate from the Biermann battery only, while the MHD dynamo (the first term of the rhs of Eq. (3)) does not operate in this case. The Ohmic term describes the decay of magnetic field due to magnetic diffusivity.

In our studies, we use a single fluid description with two layers of slightly different density. Conceptually, the $\mathrm{KH}$ instability requires only an interface with velocity shear, while density does not have to be necessarily different for the two layers. The linear stability analysis for infinitesimal perturbations $y=y_{0}+f(x, t)$ with $f(x, t) \propto \exp (\sigma t+i k x)$ for the inviscid case predicts the KH instability growth rate as ${ }^{34}$

$$
\sigma=\frac{2 \sqrt{\Theta}}{1+\Theta} k U_{0}-\frac{\Theta-1}{\Theta+1} k U_{0} i
$$

where $\Theta=\rho_{\max } / \rho_{\text {min }}>1$ is the density ratio of the two layers, $k$ is the perturbation wavenumber, and the plasma in the two layers moves initially with velocities $u_{x}= \pm U_{0}$. The dispersion relation (9) includes both real and imaginary parts, although the former term is much larger than the latter one in our simulations (see below), $\operatorname{Im}[\sigma] / \operatorname{Re}[\sigma] \approx 0.02$. As we can see, the largest growth rate corresponds to the case of equal densities of the two layers, $\Theta=1$, which however, eliminates the magnetic field generation in incompressible $\mathrm{KH}$ flow. The Biermann battery term generates magnetic field when direction of the pressure and density gradients are different. In compressible flows, the density gradient may arise through plasma compression and scales as $\nabla \rho \sim$ $\nabla p / c_{s}^{2}$ being proportional to the Mach number square, $\propto$ $M a^{2}$. Within this paper, we are interested in the almost incompressible case $M a \ll 1$; compressibility effects will be discussed in further works. Then in the case of uniform initial density in slightly compressible flows, the Biermann battery effects become as weak as $M a^{2} \ll 1$. For this reason, in the present work, we use slightly nonuniform density in the two counter-flowing layers, $\Theta=1.02$, thus ensuring a powerful $\mathrm{KH}$ instability and a finite density gradient at the interface. A higher density ratio leads to larger gradients and a stronger effect of the Biermann battery, even though it reduces the $\mathrm{KH}$ instability strength. In addition, we solve the entropy equation, Eq. (4), which may also be considered as an equation for temperature; it is responsible for the obliqueness between pressure and density gradients. Then, the initial structure of the transitional region between two counterflowing plasma layers is specified as

$$
u_{x} / c_{\mathrm{s} 0}=0.01 \tanh (y / d)=\ln \rho / \rho_{0}=-s / c_{P},
$$

where $d$ is the interface thickness. The transverse velocity component is set to zero initially together with all components of the magnetic field, B. The initial entropy distribution is computed in a way to have constant pressure in the whole domain, so that initial temperature is inversely proportional to the density. In order to trigger the instability, we add velocity perturbations to the initial flow, Eq. (10). We use two types of initial perturbations: either the white noise or sinusoidal perturbations for $y$ component of the velocity field.

\section{Parameters set}

The MHD description of the $\mathrm{KH}$ instability involves a number of parameters controlling the magnetic and velocity fields evolution. The whole parameter set in dimensionless form with the typical values used in our simulations is listed below

$$
\begin{aligned}
\mathrm{Ma} & =U_{0} / c_{s}=0.01, \\
\operatorname{Re} & =U_{0} L / \nu \approx 10^{3}, \\
\operatorname{Re}_{M} & =U_{0} L / \eta=\operatorname{Re}, \\
\operatorname{Pr} & =\nu / \kappa=1, \\
\Theta & =1.02,
\end{aligned}
$$

which are the Mach, Prandtl, and Reynolds numbers, the density ratio, and the magnetic Reynold's number, respectively. Here, $\kappa=K / \rho c_{P}$ is the thermal diffusivity. From the hydrodynamic point of view, the main $\mathrm{KH}$ instability parameters are the Mach and Reynolds numbers. The Mach number quantifies the compressibility effect; it also characterizes the time scale of the process relative to the acoustic time scale. In this paper, we use a rather small value of the Mach number, representing an almost incompressible flow. The Reynold's number determines the smallest length scale accessible and is also a limiting factor from a numerical point of view because, at high Reynold's numbers, the flow becomes turbulent and requires subtle resolution. In order to have reliable results for a turbulent flow, one has to resolve the Kolmogorov length scale, which increases dramatically the numerical resources demanded for the study. The Prandtl number characterizes the relative role of viscous and thermal effects of the flow. For the sake of numerical stability, the Prandtl number is always set to unity in our simulations. The density ratio of the two layers determines the growth rate of the perturbations in the linear stage. In the RT experiments, the density ratio may reach several hundreds for ICF conditions, posing an obstacle for numerical simulations. At the same time, the density ratio was quite moderate for the $\mathrm{KH}$ experiments at the OMEGA laser facility being comparable 
to unity ${ }^{8-12}$ In this paper, we also use moderate values of this factor slightly above unity, so we can use density for visualizing the hydrodynamic process.

In a magnetized plasma, the magnetic Reynolds number is also of key importance, as it characterizes decay of the magnetic field due to finite plasma conductivity. For the sake of numerical stability we keep the magnetic Reynolds number equal to the flow Reynolds number. Typical value of the flow Reynolds number for the ICF plasma, as well as for the KH instability experiments varies within $10^{4}-10^{6,8,27}$ however, in simulations the Reynolds number is always limited by the numerical resolution. The magnetic Reynolds number was estimated as $R e_{M} \sim 10^{3}$ for the ICF conditions, ${ }^{27}$ which we use in our simulations. The case of equal magnetic and viscous Reynolds numbers is also the most representative from the fundamental point of view for the studies of the baroclinic magnetic field generation, since it makes the equations for the magnetic field and vorticity evolution mathematically identical, see Eqs. (12) and (13) below. As a matter of fact, for plasma density $\rho=0.1 \mathrm{~g} \mathrm{~cm}^{-3}$ and temperature $T=0.6 \mathrm{keV}$, the kinematic viscosity and the magnetic diffusivity become the same. Such plasma parameters can be met between the ablation surface, where $T \sim 10 \mathrm{~K}$ and $R e_{M}$ $\ll R e$ and the critical surface, where $T \sim 10 \mathrm{keV}$ and $R e_{M}>R e$.

The scaled hydrodynamic parameters listed above may influence the magnetic field generation in a critical way. For example, flow compressibility is expected to affect the magnetic field evolution. The density gradient plays a governing role for the magnitude of the generated magnetic field, so that the density ratio becomes an important parameter for proper quantitative estimates. Detailed investigation of how the compressibility and the density ratio effect the instability evolution and generation of magnetic field requires separate thorough study which is beyond the scope of the present work.

In this paper, we focus on the most universal features of the magnetic field generation and its further evolution due to the KH instability. For this reason, we keep all the parameters fixed for all the simulations, using a moderate value for the Reynolds number, $\operatorname{Re} \approx 10^{3}$, to avoid a strongly turbulent flow. However, the chosen value for the Reynolds number is not too low either, so as to avoid fast viscous damping of the $\mathrm{KH}$ instability.

\section{RESULTS}

In our simulations, several stages in the $\mathrm{KH}$ instability development may be distinguished. At the linear stage, all perturbed values grow exponentially in time in agreement with the dispersion relation Eq. (9) including the sinusoidally shaped interface between the layers. As the perturbation amplitude grows, the nonlinear effects become important and a number of smaller vortices are formed, see the upper panel of Fig. 1. The small vortices interact with each other, which leads to vortex coalescence, until they merge into a single vortex of the largest possible size allowed by the system geometry. In order to observe such interacting vortices, we have performed a simulation with $\mathrm{Re}=2 \cdot 10^{3}$; the
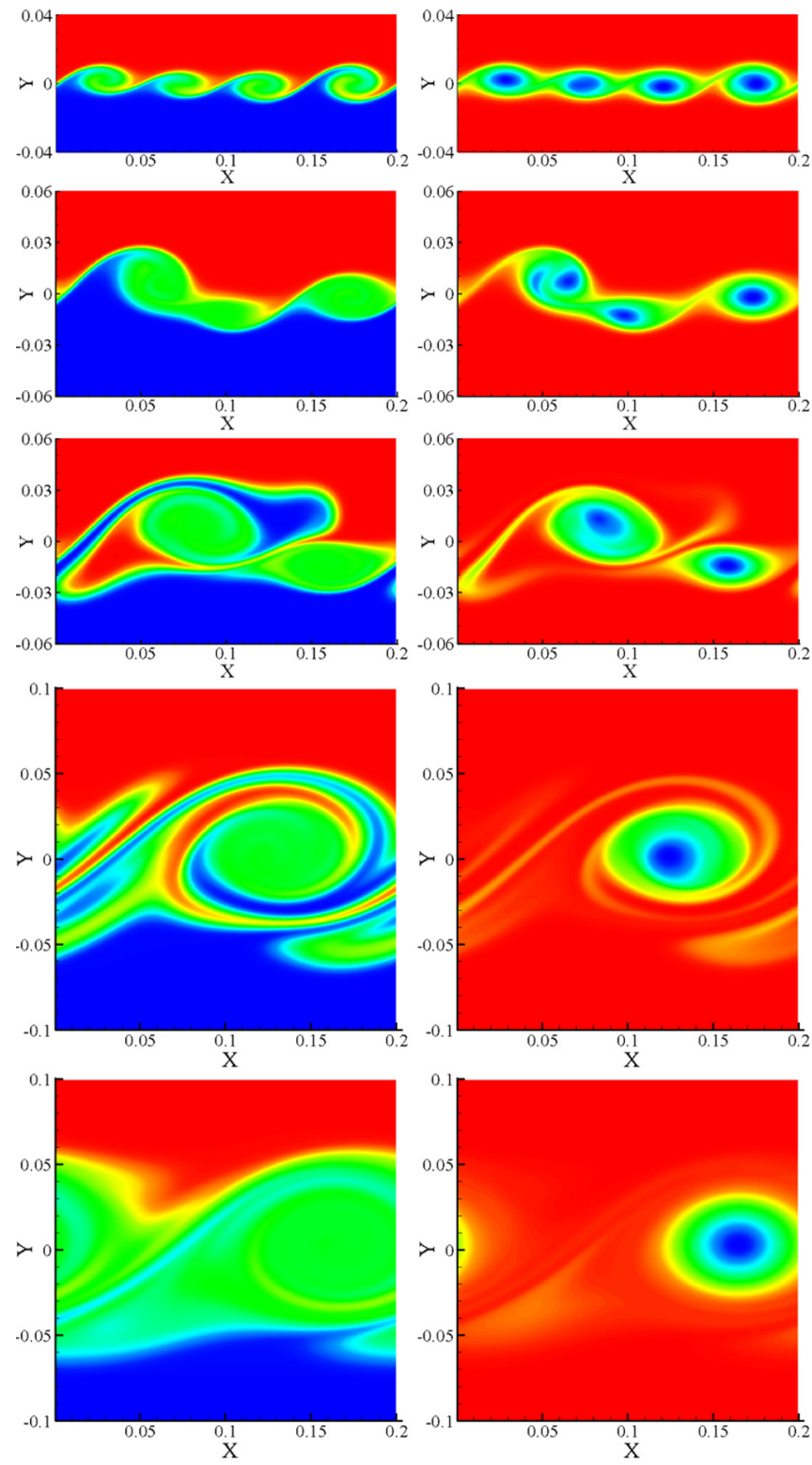

FIG. 1. Density (left panel) and vorticity (right panel) evolutions for the white noise simulation with $\operatorname{Re}=2000$ for time instants $t=2 \tau_{0} ; 3 \tau_{0} ; 4.5 \tau_{0}$; $7.5 \tau_{0} ; 12 \tau_{0}$.

corresponding sequences of density and vorticity are demonstrated in Fig. 1. After that, the large-scale vortex starts decaying due to viscosity if no external forcing is applied to support the vorticity. We can also observe a minor drift of the vortex core due to non-zero $\operatorname{Im}[\sigma]$ in Eq. (9). In the case of high Reynold's numbers, the third stage may turn into turbulent mixing of the flows leading to isotropic turbulence as the final outcome of the $\mathrm{KH}$ instability. In this paper, we consider the whole process of the instability development, though paying particular attention to the relatively early stages with no turbulence generation. In all the simulations presented below, we use a smaller Reynolds number $\left(\operatorname{Re}=10^{3}\right)$, in order to avoid possible flow turbulization and to ensure proper resolution. Here, we point out that the vortex coalescence, although common for the nonlinear $\mathrm{KH}$ instability studies, has not been observed in the Omega laser facility experiments, ${ }^{9-12}$ because of minor interaction of the 

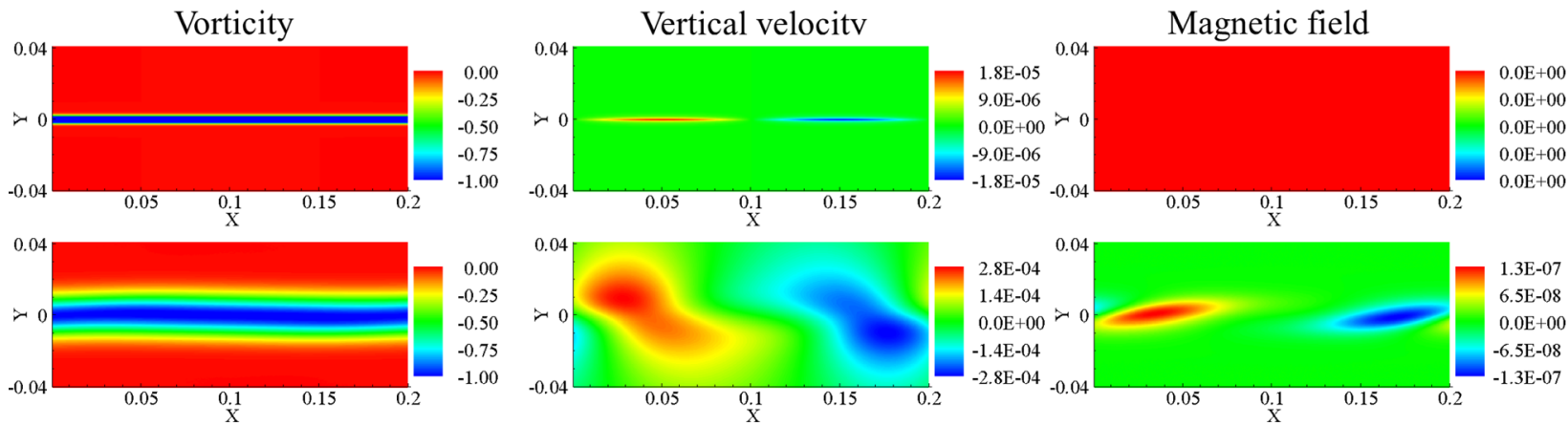

FIG. 2. Vorticity field (left), vertical velocity component $u_{y}$ (middle), and magnetic field (right) at initial moment (upper panel) and at the linear stage, at $t=3 \tau_{0}$ for a single mode simulation.

KH-generated vortices due to strong density difference between the plastic and the foam used in the set-up. The strongly nonlinear vortices observed in Refs. 9-12 had the same characteristic wavelength $\sim 400 \mu \mathrm{m}$ as the initial perturbations. The experimental data showed the complete evolution of distinct eddies from vortex formation to apparent turbulent break-up in the span of about $75 \mathrm{~ns}$ similar to the first panel of our Fig. 1. No further interaction of the KHvortices has been observed, which may be partly explained by the limited time of the $\mathrm{KH}$ instability development and other complications of the experiments. ${ }^{9-12}$

Focusing on the magnetic field generation, we naturally expect the magnetic field to have a similar structure as the flow vorticity similar to the RT-related studies. ${ }^{27-29}$ Analytically, the evolution of vorticity and the magnetic field are described by equations of the same mathematic form; by taking curl of Eqs. (2) and (3), one ends up with

$$
\begin{gathered}
\frac{\partial \mathbf{B}}{\partial t}=\nabla \times(\mathbf{u} \times \mathbf{B})-\beta \frac{\nabla \rho}{\rho^{2}} \times \nabla p+\eta \nabla^{2} \mathbf{B}, \\
\frac{\partial \boldsymbol{\omega}}{\partial t}=\nabla \times(\mathbf{u} \times \boldsymbol{\omega})+\frac{\nabla \rho}{\rho^{2}} \times \nabla p+\nu \nabla^{2} \boldsymbol{\omega},
\end{gathered}
$$

so that the equations Eqs. (12) and (13) become identical for $\mathrm{Re}=\mathrm{Re}_{m}$. Based on this similarity, one might be tempted to deduce a simple relation between the magnetic field and the flow vorticity as $B=\beta \omega$. In fact, such a relation has been demonstrated theoretically and numerically in the simulations of the RT instability in magnetized plasma. ${ }^{26-29}$ However, as has been warned already by Kulsrud, ${ }^{26}$ the simple relation $B=\beta \omega$ holds only assuming identical (e.g., zero) initial conditions for both the magnetic and vorticity fields.

By contrast, in our simulations, the generated magnetic field has significantly different structure as compared to the vorticity, see Figs. 2-4 and 8. This difference stems primarily from different initial conditions for these two quantities in our simulations; the different initial conditions are expected to be rather common for the $\mathrm{KH}$ plasma experiments. $^{9-12}$ At the initial time instant, we take zero magnetic field everywhere in the domain, while vorticity has inevitably a certain non-zero distribution due to the initial velocity profile forming the two counter-flows; see Fig. 2. As a result of this difference in the initial conditions, the magnetic field evolution is mostly governed by the second term on the right hand side of Eq. (12), while the first term can be neglected.
In the case of the vorticity equation, Eq. (13), the situation is the opposite with the first term dominating over the baroclinic one, which may be demonstrated by linearizing Eqs. (12) and (13). For the sake of simplicity, we consider the case of incompressible inviscid/non-resistive plasma flow; then, Eqs. (12) and (13) are rewritten as

$$
\begin{gathered}
\frac{\partial B_{z}}{\partial t}=-U_{x} \frac{\partial B_{z}}{\partial x}-\frac{\beta}{\rho_{0}^{2}} \frac{\partial \rho_{0}}{\partial y} \frac{\partial p}{\partial x} \\
\frac{\partial \omega_{z}}{\partial t}=-U_{x} \frac{\partial \omega_{z}}{\partial x}+\frac{\partial^{2} U_{x}}{\partial^{2} y} u_{y}+\frac{1}{\rho_{0}^{2}} \frac{\partial \rho_{0}}{\partial y} \frac{\partial p}{\partial x},
\end{gathered}
$$

where $U_{x}$ and $\rho_{0}$ stand for initial distribution of velocity and density, respectively, see Eq. (10). The latter involves small, but finite, width $d$ of the interface between the two components, and we take $d \sim 10^{-3}$ at the relatively early instability stages. The linearized form, Eqs. (14) and (15), reveals the difference between the magnetic field and vorticity equations, which has not been obvious in their original form, Eqs. (12) and (13). Different terms of Eqs. (14) and (15) can be estimated with respect to the orders of the small dimensionless width $d$ of the transitional region as

$$
\begin{gathered}
\frac{\partial B_{z}}{\partial t}=O(1) B_{z}+O(1 / d) p \\
\frac{\partial \omega_{z}}{\partial t}=O(1) \omega_{z}+O\left(1 / d^{2}\right) u_{y}+O(1 / d) p
\end{gathered}
$$

Thus, the magnetic field evolves mostly due to the Biermann battery term (second term in Eq. (16)), while the convective term may be neglected. On the other hand, the convective constituent in the vorticity equation has two terms; the second term stems from the initial vorticity distribution and dominates for the vorticity evolution. The order of magnitude analysis, Eqs. (16) and (17), of the magnetic field and vorticity equations, Eqs. (12) and (13), clarifies the evolution difference in these two key characteristics of the MHD KH instability.

We use two types of initial perturbations corresponding either to sinusoidal modes or white noise.

\section{Single-mode large scale perturbations}

Initial conditions in the form of single-mode large scale perturbations allow accurate investigation and thorough 

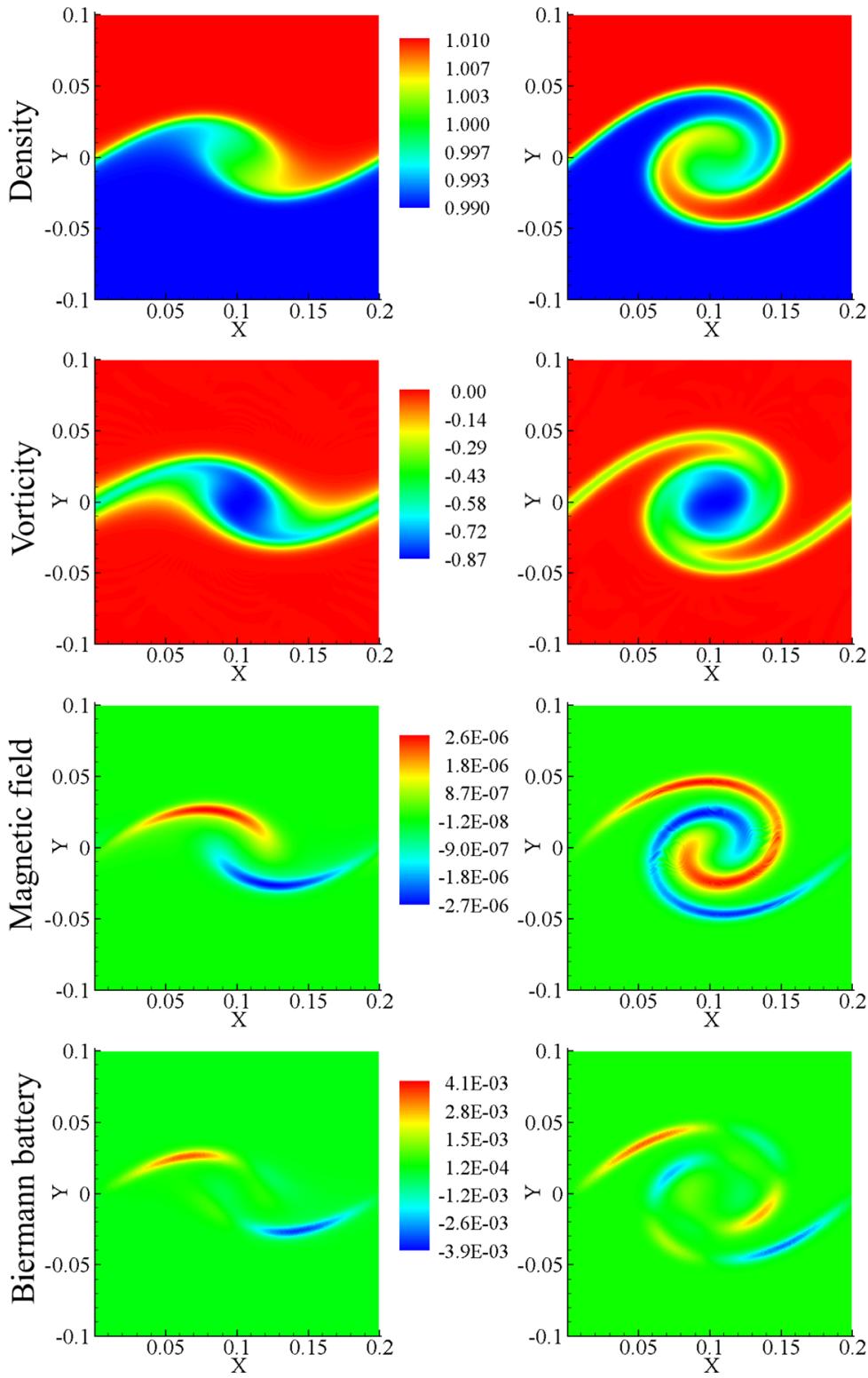
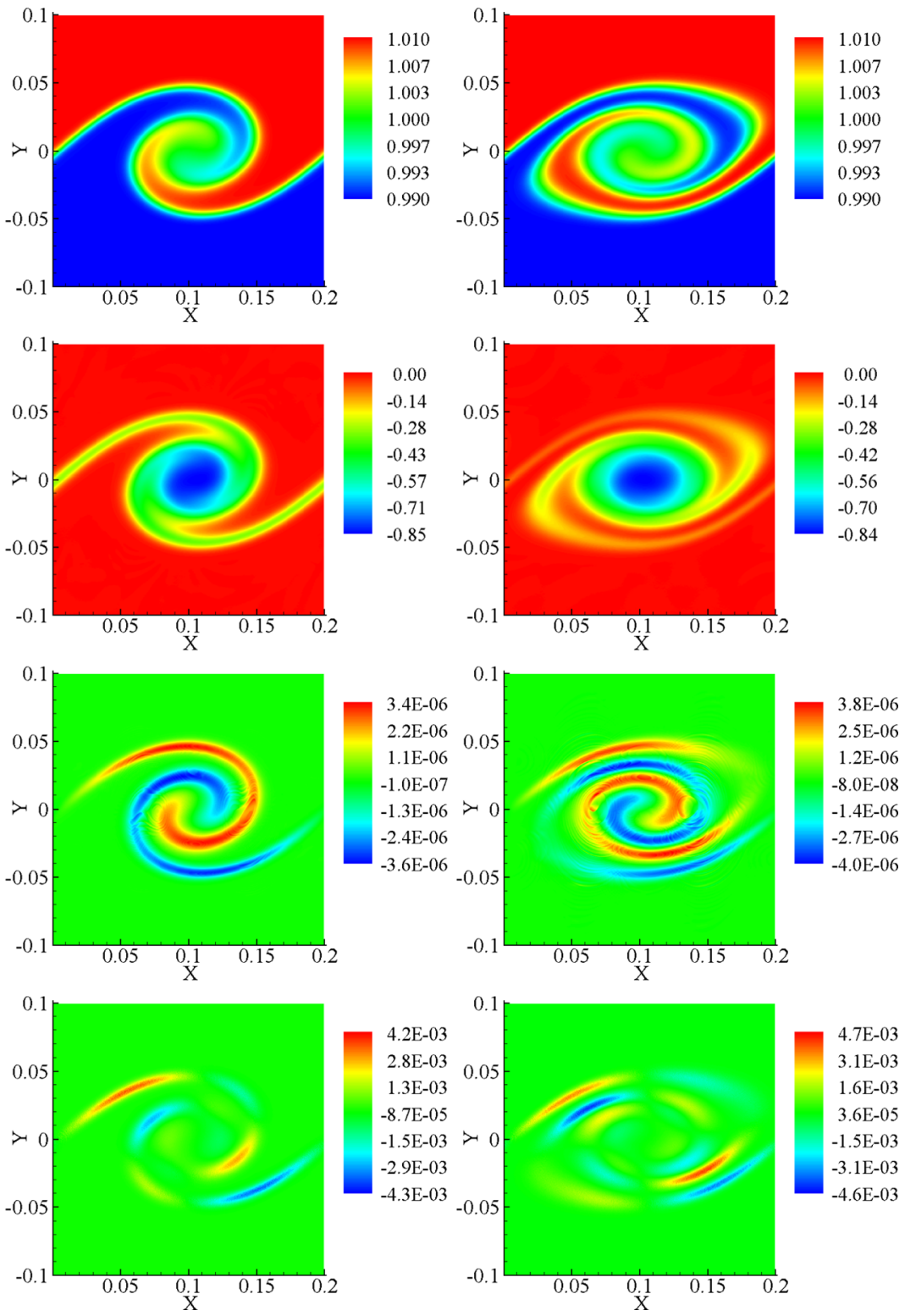

FIG. 3. Density, vorticity, magnetic field, and the Biermann battery term at $t=5 \tau_{0}, 6 \tau_{0}, 7 \tau_{0}$, respectively (left to right).

understanding of the $\mathrm{KH}$ instability at the relatively early stages. The initial transverse velocity perturbation represents a mode of a largest possible wavelength allowed by the problem geometry with the amplitude exponentially decaying to the outer walls

$$
u_{y}=\tilde{u}_{y} \sin (k x) \exp (-|y| / w)
$$

where $k=k_{0}=2 \pi / D$ is the perturbation wavenumber, $D$ is the length of the domain, and $w$ is the interface width. The instability development at the linear stage is demonstrated in Fig. 2 together with the generated magnetic field. The large wavelength of the perturbation mode leads to the slowest growth rate of the instability, so that after three turnover times, $t=3 \tau_{0}$, with $\tau_{0}=L_{0} / U_{0}$, the perturbations may be still
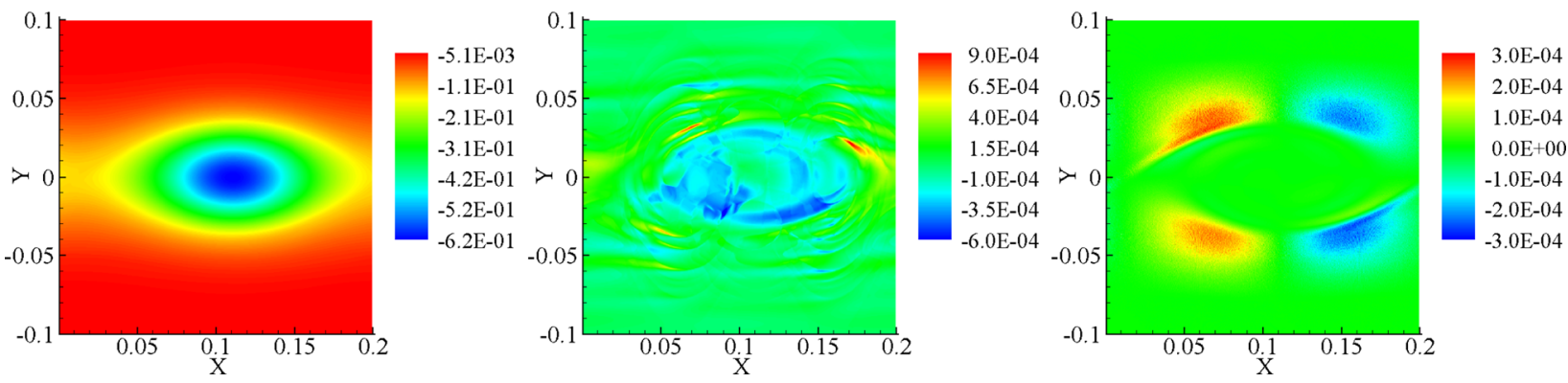

FIG. 4. Distribution of vorticity (left), magnetic field (middle), and baroclinic term (right) in the very later stage of the KH instability, $t=20 \tau_{0}$. 

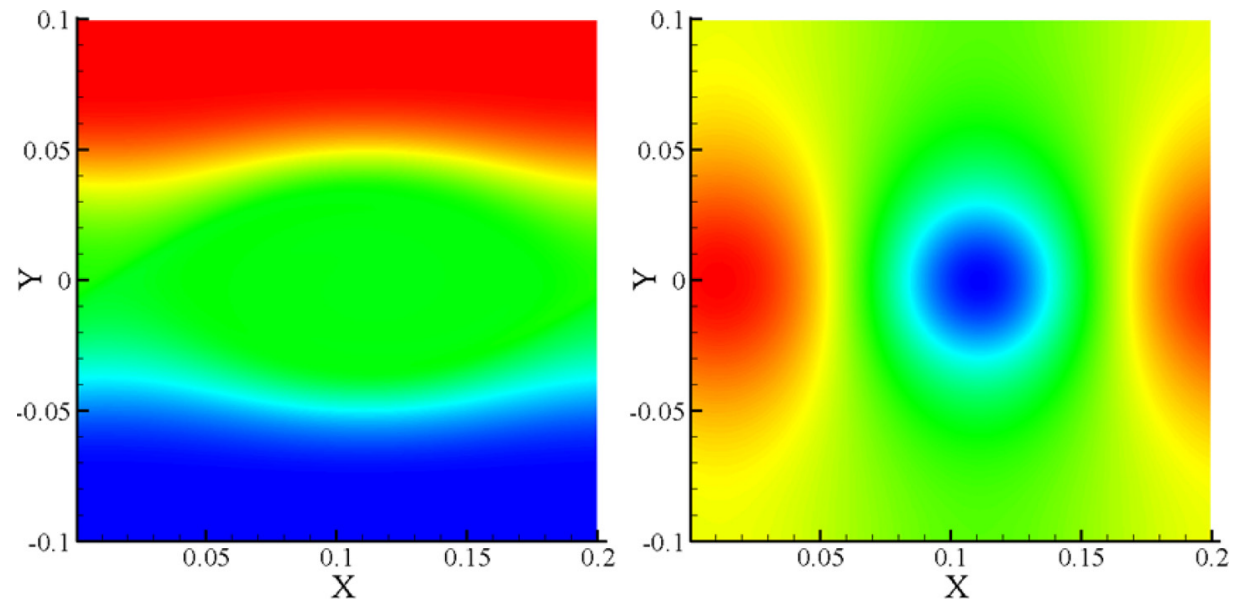

FIG. 5. Distributions of density (left) and pressure (right) corresponding to the previous picture. treated as linear. The vorticity distribution is modified due to the interface bending, with the vorticity pattern already much wider than the original interface. During the linear instability stage, two regions of the opposite magnetic field direction have been formed near the humps/troughs of the distorted interface. Figure 2 demonstrates clearly that the generated magnetic field structure is quite different from the vorticity field. It should be noted that the color maps for vorticity and magnetic field are also different: Initially, the vorticity is almost zero in the whole domain (shown as red), while in the middle region, it reaches a certain negative value, depicted in dark blue. The $z$ component of the magnetic field takes negative (blue) and positive (red) values with zero background shown by green; this coloring is also used for all other figures.

Figure 3 presents the distributions of density, vorticity, the generated magnetic field, and the Biermann battery term at three time instants at the strongly nonlinear stage of the instability development, with wave breaking and formation of a single large vortex characteristic for the $\mathrm{KH}$ instability. At this stage, the two fluids start mixing, which produces additional regions of high density gradients and leads to a specific spiral structure of the magnetic field. The baroclinic term structure in Fig. 3 is also swirling, but it demonstrates an interesting tendency as compared to the magnetic field spiral. Both structures are quite similar at the relatively early stages, e.g., at $t / \tau_{0}=5$, for which the last term in Eq. (12) is dominating

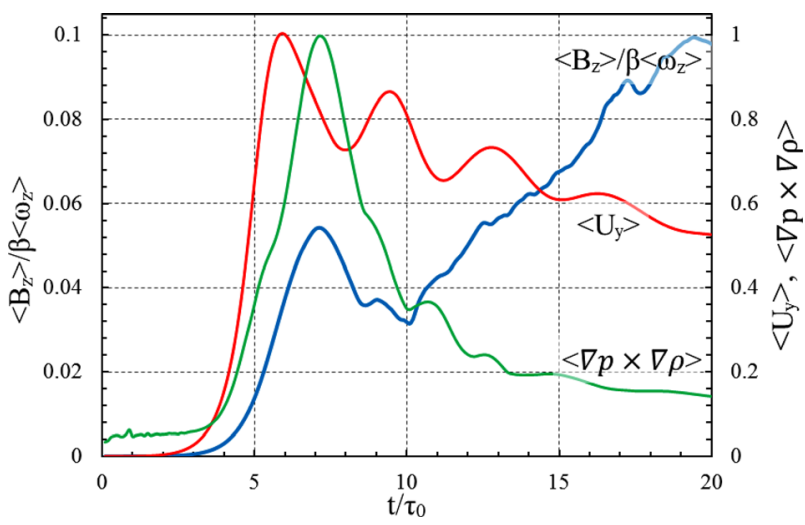

FIG. 6. Time evolution of the scaled averaged quantities representing magnetic field, baroclinic term, and velocity $u_{y}$. and governs the magnetic field evolution. Still, the structures become different somewhat later, e.g., at $t / \tau_{0}=6 ; 7$, with the baroclinic term spiral breaking up into "islands" of opposite sign because of the interface of high density gradients rollingup into an eddy. The spiral of the magnetic field, however, remains continuous keeping the initial signs due to convective term in Eq. (12), which results in the swirling magnetic field structure presented in Fig. 3 for $t / \tau_{0}=6 ; 7$. Still, the baroclinic term remains dominating in the magnetic field generation. To make sure that the spiral waves do not stem from poor resolution, additional simulations with higher resolution have been performed. These runs suggest that the spiral structures originate from physical effects, e.g., the interference of several magnetic field sources located in different places or a secondary instability leading to spiral patterns as one can find in nonlinear physics. ${ }^{35-37}$

At a much later stage illustrated in Figs. 4 and 5, the $\mathrm{KH}$ instability pattern exhibits a well-developed single vortex, which corresponds to circular distribution of the flow vorticity and decays slowly due to viscosity. The elongated vortex shape in Fig. 4 is a result of the initial flow influence, which stretches the vortex in line with the flow. At that stage, the magnetic field structure is quite different from both the vorticity and the baroclinic term structures; the magnetic field pattern demonstrates a complex interplay of the field generation by the Biermann battery term and convective transfer by

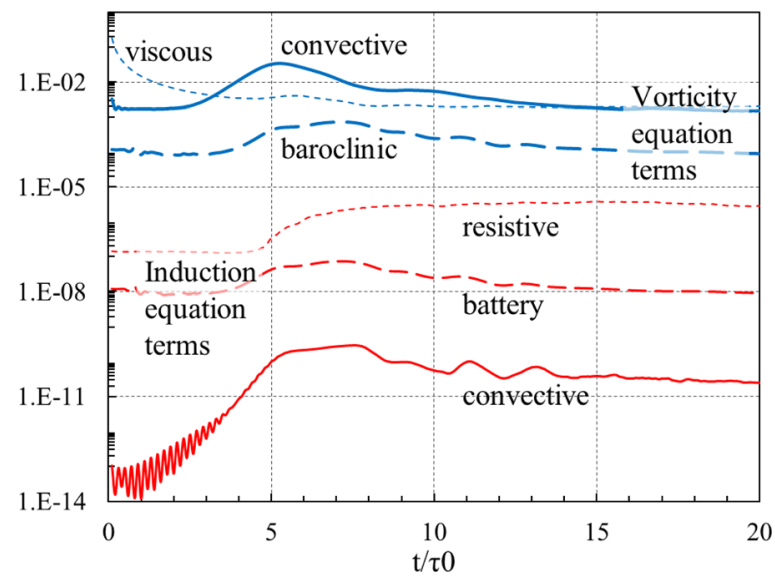

FIG. 7. Relative role of each of the terms of the vorticity equation, Eq. (13), and the induction equation, Eq. (12), shown by means of the RMS values. 

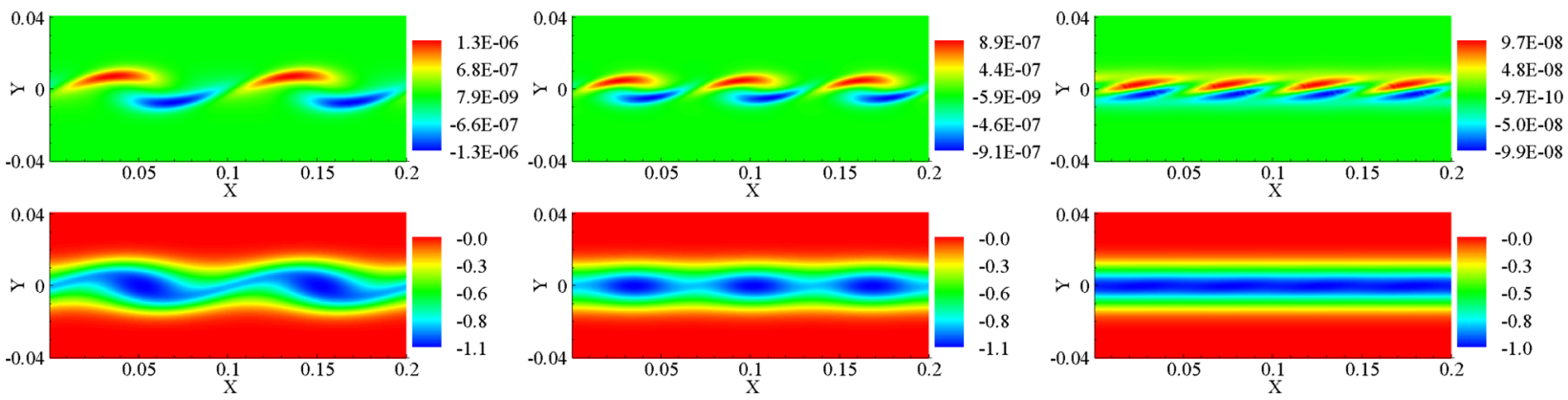

FIG. 8. Magnetic field (upper panel) and vorticity (lower panel) at time instant $t=3 \tau_{0}$ for initial perturbation of $k=2 k_{0} ; 3 k_{0} ; 4 k_{0}$.

the flow. The magnetic field pattern with a region of negative magnetic field dominating in the vortex center in Fig. 4 is not properly understood yet and demands further investigation well beyond the scope of the present paper. The baroclinic term in Fig. 4 originates in the spatial distributions of density and pressure in the flow, Fig. 5, which demonstrate a mixing layer of an almost homogeneous density inside the vortex. Still, the upper and lower parts of the domain are filled with unmixed components providing noticeable density gradients on the outer sides of the vortex core. The pressure distribution in the vortex is governed by the hydrodynamical contribution, as the flow remains isobaric in total. There is a certain pressure minimum in the vortex core due to flow compressibility, and the pressure variations do not exceed the $\sim \mathrm{Ma}^{2}$ estimate.

Another interesting feature, which is observed in all the simulations, is that the magnetic field strength keeps growing during the whole process, as shown in Fig. 6, even when the vortex strength started decaying. This specific magnetic field behavior results from the Biermann battery term structure, i.e., due to different directions of the density and pressure gradients in the flow. As shown in Fig. 4, at the late stages of the KH instability, the Biermann battery term acquires a peculiar structure with small, but finite magnitude, which remains almost constant and supports the continuous magnetic field growth. In Fig. 6, we also plot the time evolution of the averaged values for the baroclinic term and velocity $u_{y}$. In order to present all the values in one plot, velocity and baroclinic terms have been scaled to their maximal values; the magnetic field is scaled by $\beta\left\langle\omega_{z}\right\rangle$. Here, $\left\langle\omega_{z}\right\rangle$ is the mean vorticity at the end of the run at $t=20 \tau_{0}$. Within such a scaling, the value $\left\langle B_{z}\right\rangle / \beta\left\langle\omega_{z}\right\rangle$ would be unity, provided that $\omega_{z}$ was created solely by the baroclinic term, which, however, is not the case for the present study. Remarkably that, even though most of the contribution to $\omega_{z}$ comes from the shear flow, this ratio still reaches values of about 0.1 . During the relatively early stage of the instability development, $t / \tau_{0}<5$, all quantities grow exponentially in a similar way, and the non-zero Biermann battery term due to sharp density gradients is coupled to the velocity perturbations. As the $\mathrm{KH}$ instability develops, the vortex velocity reaches it maximal value at $t \approx 6 \tau_{0}$ and starts decaying afterwards. The baroclinic term shows similar behavior, although it decays to some small finite level. This value is a few orders of magnitude smaller than the initial vorticity, so that its contribution to vorticity production is minor and cannot overcome the viscous decay. The baroclinic term is, however, large enough to sustain the noticeable magnetic field growth, as shown in Fig. 6.

In Fig. 7, we compare the RMS (root-mean-square) averaged values of every term in the vorticity and induction equations, Eqs. (11) and (12). We point out that the respective terms represent derivatives of different orders and hence their relative strength depends quite strongly on the problem length scale and the Reynolds number. The dissipative terms involve the third order derivatives, which make them dominating at the smallest length scales at moderate values of the Reynolds number as shown in Fig. 7. Nevertheless, the convective term of the vorticity equation exceeds the baroclinic term by more than an order of magnitude in Fig. 7, which agrees with our previous estimates, Eqs. (16) and (17). On the contrary, the Biermann battery term dominates over the convective term in the induction equation, which, again, supports our order-of-magnitude analysis.

\section{Several-mode small-scale perturbations}

The single large-scale mode simulations presented above demonstrate basic features of the plasma KH instability including the baroclinic magnetic field generation, but they do not involve vortex interaction. To study the evolution and interaction of multiple vortices in the $\mathrm{KH}$ instability, we have performed several simulation runs with larger wavenumbers of initial perturbations, $k / k_{0}=2,3,4$. According to the dispersion relation, Eq. (9), small vortices with larger wavenumbers grow faster at the linear stage than a single vortex of the largest possible size. At the relatively early stages of the instability development, the growth of individual vortices resembles the evolution of a single vortex described in "Single-mode large scale perturbations" section, although at a shorter time, $\sim(U k)^{-1}$, with faster magnetic field generation. The early stages of vortex formation are depicted in Fig. 8 for the magnetic field and vorticity distributions. The vortices can be seen both in magnetic field and vorticity, although the magnetic field represents the vortex locations more clearly, while vorticity has a very smooth profile. For relatively short wavelength perturbations with $k / k_{0}=4$, the perturbation vorticity pattern at $t=3 \tau_{0}$ is still obscured by the original vorticity of the transitional layer, but the magnetic field structure is clearly seen.

Although this paper is devoted mostly to the nonlinear $\mathrm{KH}$ instability stage and to the magnetic field generation, it 


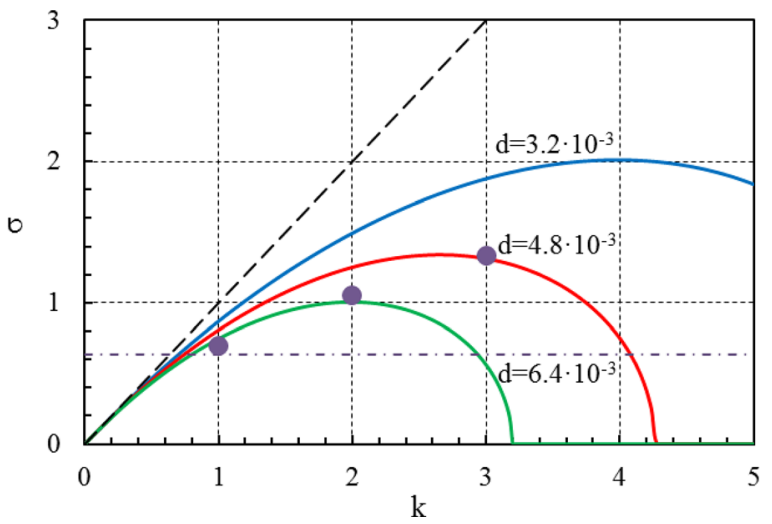

FIG. 9. Dispersion relation for different width of velocity profile, dashed line corresponds to classical expression $\sigma=k U_{0}$, dots represent numerical results, dash-dotted line stands for white noise case.

is interesting to check the linear growth rate obtained numerically and compare it to the theoretical predictions. It appears that the instability growth rates obtained in our simulations for different wavenumbers are considerably less than those predicted by Eq. (9), which, however, has been derived for the simplified case of an infinitely thin interface between the moving plasma layers. Calculating the instability growth rate for the present geometry more accurately, by adopting almost constant density in the whole domain, and the velocity distribution in the form

$$
U_{x}= \begin{cases}U_{0}, & y>d \\ U_{0} y / d, & -d<y<d \\ -U, & y<-d,\end{cases}
$$

we find the dispersion relation as

$$
\sigma=U_{0} k \sqrt{-1+\frac{1}{k d}-\frac{1-\exp (-4 k d)}{4 k^{2} d^{2}}}
$$

In the case of small $d$, this expression can be reduced to $\sigma=k U_{0}(1-4 k d / 3)$, which demonstrates stabilization of the $\mathrm{KH}$ instability due to finite transition width in the velocity profile. In Fig. 9, we have plotted the growth rate for different widths $d$ together with our simulation results. The plots exhibit a reduction of the instability growth rate due to the finite width of the transitional layer leading to complete stabilization for sufficiently short perturbation wavelengths. The numerical simulation points lay noticeably lower than the classical expression (presented by the dashed line). However, they show fair agreement with the more accurate dispersion relation for $d=4.5 \cdot 10^{-3}$, which is comparable with the width value at the early stage in our simulations.

In order to get a better insight into length scale dependence, we remind that the interface width is mostly determined by the thermal conduction. As the later is a transport process of diffusion nature, so the interface width increases with time as $d \sim \sqrt{t}$ and requires respective corrections to the interface width employed in Eq. (18). Figure 10 presents evolution of the maximal vertical velocity during early stage of the $\mathrm{KH}$ instability obtained in the numerical simulations and taken from the analytical theory with corrections to the interface width. The solid lines represent numerical results from our simulation for different wavenumbers; the dashed lines stand for Eq. (18), where $d=d_{0} \sqrt{t}$. In spite of the simplified theoretical model, we do observe very good agreement of our numerical results with the analytical estimates. More accurate analysis should include smooth transition in velocity profile together with a certain density profile.

At the later stage of the process, we observe the interaction of vortices as illustrated in Fig. 1, which may be regarded as a transient in the evolution from multiple smallscale vortices to a single large vortex of a maximal possible size. These smaller vortices at the KH-unstable interface represent a mixing layer rather than separately spinning eddies; this effect becomes obvious for perturbations of high wavenumbers. Merging of vortices takes place in a mixing layer in a smooth manner; we demonstrate it by showing the density and vorticity for the case $k=2 k_{0}$ in Fig. 11 . At the final stage of a single vortex, all the quantities have similar structures to those depicted in Figs. 4 and 5.

\section{White noise perturbation}

Finally, we consider the $\mathrm{KH}$ instability evolution and the magnetic field generation for the white noise perturbations at the interface between two counter-flowing plasma
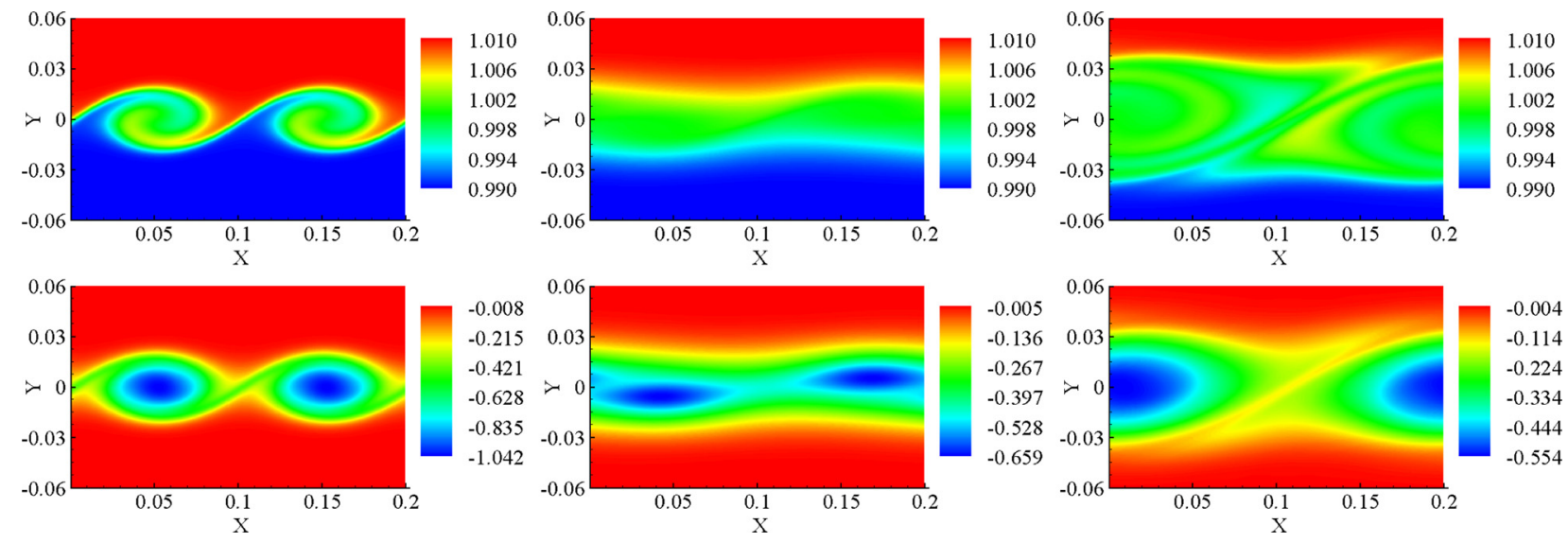

FIG. 10. Evolution of maximal value of normal to interface velocity for different wavenumbers from our DNS (solid lines); analytical predictions due to Eq. (18) together with $d=d_{0} \sqrt{t}$ are shown as dashed lines. 


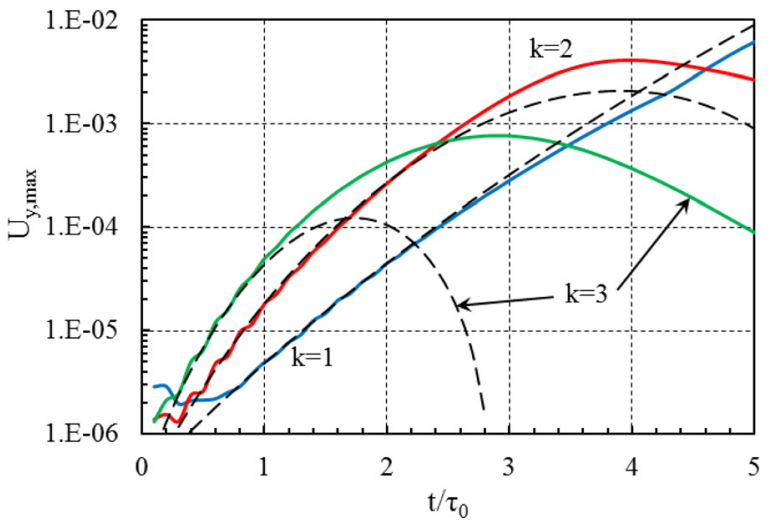

FIG. 11. Density (upper panel) and vorticity (lower panel) evolutions during merging of two eddies for $k=2 k_{0}$, time moments correspond to $t / \tau_{0}=4,10$, and 15 .

layers. In that case, the initial vortex size is not prescribed, even for the early instability stage, and the system is allowed to choose its own characteristic wavelength. In the theoretical case of an infinitely thin interface between the plasma flows, the dispersion relation Eq. (9) does not imply any suppression in the high wavenumber range, ${ }^{34}$ which implies fast perturbation growth of the wavelength as small as possible. In the present simulations, however, the interface has a finite thickness, and the dispersion relation has to be corrected as Eq. (18) with the maximal growth rate expected for perturbations of $k d \sim 1$, with the respective size of magnetic field perturbations comparable to the interface width $d$ at the early $\mathrm{KH}$ instability stage. In agreement with these expectations, Fig. 12 shows characteristic size of the magnetic field spots about $d$ for $t / \tau_{0}=1$; regions of different sign of the magnetic
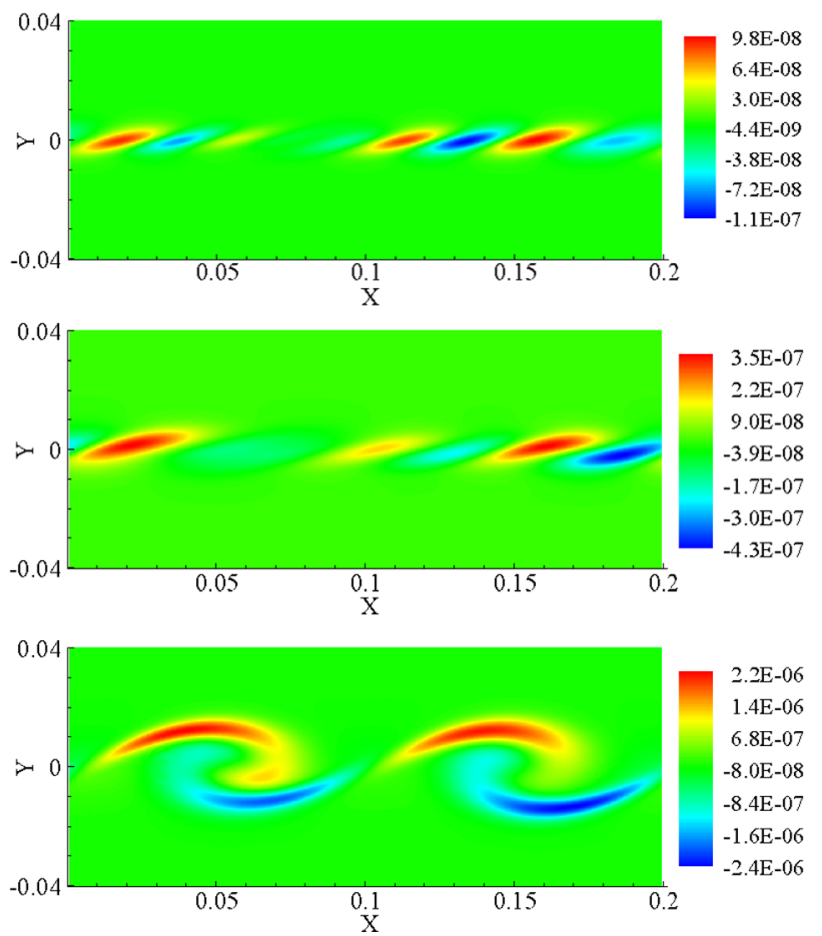

FIG. 12. Magnetic field evolution in the simulation with the white noise initial perturbation at time instants $t=\tau_{0}$ (top), $t=2 \tau_{0}$ (middle), and $t=4 \tau_{0}$ (bottom).

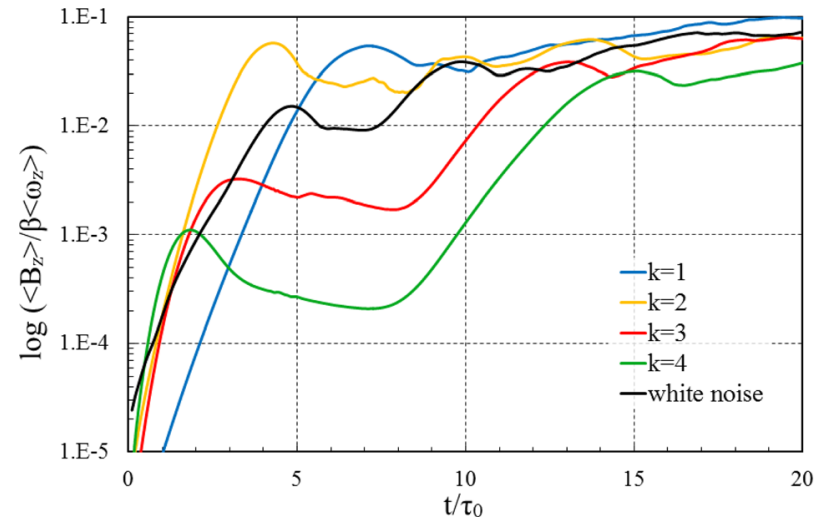

FIG. 13. Magnetic field evolution versus time for different initial perturbations in logarithmic scale.

field indicate the location and number of vortices. At later instability stages, the vortices coalesce and the magnetic field spots start merging and form eventually a spiral structure of the largest possible size. In Fig. 12, we observe four vortices at $t / \tau_{0}=1$ and only two at $t / \tau_{0}=4$. Starting from $t / \tau_{0}=4$, the instability evolution resembles the case with $k=2 k_{0}$, described in "Several-mode small-scale perturbations" section, although it evolves somewhat faster. In the simulation run with $k=2 k_{0}$, two eddies merge into a single vortex after about ten turnover times, $\sim 10 \tau_{0}$, while in the white noise run, the two eddies merge into a single one after in a time of $\sim 5 \tau_{0}$.

The scaled average magnetic field evolution for different perturbation modes is summarized in Fig. 13. Roughly speaking, the magnetic field evolution may be divided into two parts, where the first one corresponds to the exponential growth during the earlier stages of the instability, and the second part represents an almost linear growth at the later stages of the process (mind the logarithmic scales). In agreement with the linear dispersion relation, Eq. (9), the magnetic field grows faster for larger wavenumbers of the initial perturbations. Remarkably, the white noise case demonstrates relatively weaker growth at the early $\mathrm{KH}$ instability stages, because the linear instability is quite short for the white noise case involving short wavelength perturbations. In particular, Figure 12 demonstrates several small eddies for the white noise case already at $t=\tau_{0}$, but an eddy is an essentially nonlinear phenomenon. The interaction of small vortices produces a wide mixing layer in relatively short time; after that, the instability continues developing, but with a reduced growth rate, as follows from the above analysis.

The magnetic field evolution depicted in Fig. 13 has several interesting features. For all cases, except $k=k_{0}$, there is a certain plateau in the magnetic field growth. The plateau corresponds to the period when the mixing layer is formed and, hence, the evolution of the instability slows down and the baroclinic term decreases. In addition, each curve has one or several pronounced peaks, e.g., at $t=5 \tau_{0}$ and $10 \tau_{0}$ for the white noise case. These peaks correspond to the smaller vortices merging into bigger ones. Qualitatively, it may be understood as an increase of the two fluid mixing, giving birth to additional slices of different densities. This process 
produces additional areas with non-zero baroclinic term and results in the magnetic field generation at increased rate.

\section{CONCLUSIONS}

In this paper, we have investigated the KH instability in fully ionized plasmas focusing on the magnetic field generation through the Biermann battery (baroclinic) effects. As compared to the magnetic dynamo, the KH instability leads to magnetic field growth from zero, with no initial seeding. In contrast to the related works on the RT instability with the battery term, ${ }^{28,29}$ we have demonstrated that the instability generated magnetic field and vorticity structures may be quite different, even though they obey similar equations, Eqs. (12) and (13), which become mathematically identical for the Reynolds number equal the magnetic Reynolds number, $\mathrm{Re}=\mathrm{Re}_{m}$. The distinction between the magnetic field and vorticity structures in our simulations originates from intrinsically different initial conditions for these two values, which are supposed to be rather common for the KH plasma experiments. ${ }^{8-12}$ Another important finding of the present work is that the magnetic field continues to grow even after the largest vortex has been formed and started decaying. It should be mentioned too that in the present simulations, we take the flow parameters resulting in a relatively weak generated magnetic field, so that it does not affect the hydrodynamic flow. Our results demonstrate that the relation between vorticity and the magnetic field in the MHD instabilities is not as straightforward, as it was believed previously, and indicate wide prospects for future research.

\section{ACKNOWLEDGMENTS}

We thank Dhrubaditya Mitra and Matthias Rheinhardt for help and useful comments. We are also grateful to the referee for useful comments which lead to better presentation of our results and improving our understanding of the problem. Financial support from the European Research Council under the AstroDyn Research Project No. 227952, the Swedish Research Council under the Grant Nos. 621-2011-5076 and 2012-5797, as well as the Research Council of Norway under the FRINATEK Grant No. 231444 are gratefully acknowledged. We acknowledge the allocation of computing resources provided by the Swedish National Allocations Committee at the Center for Parallel Computers at the Royal Institute of Technology in Stockholm and the National Supercomputer Centers in Linköping, the High Performance Computing Center North in Umeå, and the Nordic High Performance Computing Center in Reykjavik.
${ }^{1}$ S. Chandrasekhar, Hydrodynamic and Hydromagnetic Stability (Dover Publications, New York, 1981).

${ }^{2}$ Y. Shen, J. M. Stone, and T. A. Gardiner, ApJ 653, 513 (2006).

${ }^{3}$ J. A. Barranco, ApJ 691, 907 (2009).

${ }^{4}$ C.-Y. Wang and R. A. Chevalier, ApJ 549, 1119 (2001).

${ }^{5}$ N. Suzuki, H. Takeuchi, K. Kasamatsu, M. Tsubota, and H. Saito, Phys. Rev. A 82, 063604 (2010).

${ }^{6}$ D. Kobyakov, A. Bezett, E. Lundh, M. Marklund, and V. Bychkov, Phys. Rev. A 89, 013631 (2014).

${ }^{7}$ V. Bychkov, D. Valiev, V. akkerman, and C. K. Law, Combust. Sci. Technol. 184, 1066 (2012).

${ }^{8}$ O. A. Hurricane, High Energy Density Phys. 4, 97 (2008).

${ }^{9}$ O. A. Hurricane, J. F. Hansen, H. F. Robey, B. A. Remington, M. J. Bono, E. C. Harding, R. P. Drake, and C. C. Kuranz, Phys. Plasmas 16, 056305 (2009).

${ }^{10}$ E. C. Harding, J. F. Hansen, O. A. Hurricane, R. P. Drake, H. F. Robey, C. C. Kuranz, B. A. Remington, M. J. Bono, M. J. Grosskopf, and R. S. Gillespie, Phys. Rev. Lett. 103, 045005 (2009).

${ }^{11}$ K. S. Raman, O. A. Hurricane, H.-S. Park, B. A. Remington, H. Robey, V. A. Smalyuk, R. P. Drake, C. M. Krauland, C. C. Kuranz, J. F. Hansen, and E. C. Harding, Phys. Plasmas 19, 092112 (2012).

${ }^{12}$ F. W. Doss, E. N. Loomis, L. Welser-Sherrill, J. R. Fincke, K. A. Flippo, and P. A. Keiter, Phys. Plasmas 20, 012707 (2013).

${ }^{13}$ D. Layzer, Astrophys. J. 122, 1 (1955).

${ }^{14}$ R. Betti and J. Sanz, Phys. Rev. Lett. 97, 205002 (2006).

${ }^{15}$ M. Modestov, V. Bychkov, R. Betti, and L.-E. Eriksson, Phys. Plasmas 15, 042703 (2008).

${ }^{16}$ V. Bychkov, M. Modestov, V. Akkerman, and L.-E. Eriksson, Plasma Phys. Controlled Fusion 49, B513 (2007).

${ }^{17}$ Y. Kuramitsu, Y. Sakawa, S. Dono, C. D. Gregory, S. A. Pikuz, B. Loupias, M. Koenig, J. N. Waugh, N. Woolsey, T. Morita, T. Moritaka, T. Sano, Y. Matsumoto, A. Mizuta, N. Ohnishi, and H. Takabe, Phys. Rev. Lett. 108, 195004 (2012).

${ }^{18}$ J. A. Stamper, K. Papadopoulos, R. N. Sudan, S. O. Dean, E. A. McLean, and J. M. Dawson, Phys. Rev. Lett. 26, 1012 (1971).

${ }^{19}$ K. Mima, T. Tajima, and J. N. Leboeuf, Phys. Rev. Lett. 41, 1715 (1978).

${ }^{20}$ M. G. Haines, Can. J. Physiol. 64, 912 (1986).

${ }^{21}$ J. A. Stamper, Laser Part. Beams 9, 841 (1991).

${ }^{22}$ G. A. Askat'yan, M. S. Rabinovich, A. D. Smirnova, and V. B. Studenov, JETF Lett. 5, 93 (1967).

${ }^{23}$ J. R. Rygg et al., Science 319, 1223 (2008).

${ }^{24}$ L. Gao, P. M. Nilson, I. V. Igumenschev, S. X. Hu, J. R. Davies, C. Stoeckl, M. G. Haines, D. H. Froula, R. Betti, and D. D. Meyerhofer, Phys. Rev. Lett. 109, 115001 (2012).

${ }^{25}$ M. J.-E. Manuel, C. K. Li, F. H. Seguin, J. A. Frenje, D. T. Casey, R. D. Petrasso, S. X. Hu, R. Betti, J. Hager, D. D. Meyerhofer, and V. Smalyuk, Phys. Plasmas 19, 082710 (2012).

${ }^{26}$ R. M. Kulsrud, R. Cen, J. P. Ostriker, and D. Ryu, ApJ 480, 481 (1997).

${ }^{27}$ B. Srinivasan, G. Dimonte, and X.-Z. Tang, Phys. Rev. Lett. 108, 165002 (2012).

${ }^{28}$ B. Srinivasan and X.-Z. Tang, Phys. Plasmas 19, 082703 (2012).

${ }^{29}$ F. Modica, T. Plewa, and A. Zhiglo, High Energy Density Phys. 9, 767 (2013).

${ }^{30}$ E. P. Alves, T. Grismayer, S. F. Martins, F. Fiza, R. A. Fonseca, and L. O. Silva, Astrophys. J. Lett. 746, L14 (2012).

${ }^{31}$ See http://pencil-code.googlecode.com.

${ }^{32}$ A. Brandenburg and W. Dobler, Comput. Phys. Commun. 147, 471 (2002).

${ }^{33}$ W. Zhang, A. MacFadyen, and P. Wang, Astrophys. J. 692, L40 (2009).

${ }^{34}$ T. Funada and D. D. Joseph, J. Fluid Mech. 445, 263 (2001).

${ }^{35}$ G. Jomaas and C. K. Law, Phys. Fluids 22, 124102 (2010).

${ }^{36}$ M. Cross and P. Hohenberg, Rev. Mod. Phys. 65, 851 (1993).

${ }^{37}$ V. Zykov and E. Bodenschatz, Phys. Rev. Lett. 112, 054101 (2014).

${ }^{38}$ See subsection "Diffusive error from time-stepping" under section "Numerical methods" in "Appendix" chapter in the PENCIL CODE Manual. ${ }^{31}$ 